\title{
Dynamic product diversity
}

\author{
Ramon Caminal* \\ Institut d'Anàlisi Ecònomica, CSIC, and Barcelona GSE
}

December 5, 2011

\begin{abstract}
The goal of this paper is to study the frequency of new product introductions in monopoly markets where demand is subject to transitory saturation. We focus on those types of goods for which consumers purchase at most one unit of each variety, but repeat purchases in the same product category. The model considers infinitely-lived, forward-looking consumers and firms. We show that the share of potential surplus that a monopolist is able to appropriate increases with the frequency of introduction of new products and the intensity of transitory saturation. If the latter is sufficiently strong then the rate of introduction of new products is higher than socially desirable (excessive dynamic product diversity.)

JEL Classification numbers: L12, L13

key words: transitory saturation, product diversity, repeat purchases, demand cycles
\end{abstract}

\section{Introduction}

The goal of this paper is to study the frequency of new product introductions in monopoly markets where demand is subject to transitory saturation. Most of the attention will be on those types of goods for which consumers tend to purchase just one unit of a particular variety, but engage in repeat purchases in the same product category. Examples abound in the general category of leisure goods: books, music recordings, movies, computer games, concerts, etc. In these cases the pattern of repeat purchases is closely associated to the rate at which new varieties are supplied. In this context it is natural to ask whether markets tend to introduce new varieties too quickly or too slowly with respect to some efficiency benchmark.

*I would like to thank Ricard Gil, Martin Perry, Pasquale Schiraldi, and especially Roberto Burguet for their useful comments. I am also grateful to the Spanish Ministry of Science and Innovation (ECO2011-29663), and Generalitat de Catalunya for their financial support. 
The literature on product differentiation typically considers the static tradeoff between heterogeneous consumer preferences and the fixed cost associated to the production of each variety. Salop (1979)'s circular city model or Chen and Riordan (2007)'s spokes model are popular examples of frameworks where each consumer is portrayed as buying one unit of a particular variety, and hence different varieties appeal to separate consumer groups. ${ }^{1}$

In many of the markets mentioned above, at some level of aggregation, we also observe product diversity in the standard sense. But when we focus on a particular segment of the market (for instance, romantic comedies produced by large Hollywood studios, or historical novels released by major publishing houses), we recognize that some consumers tend to purchase the same variety at a point in time, and that their taste for diversity is mostly expressed over time as new varieties become sequentially available. In fact, commercialization and consumption are highly synchronized: most purchases are typically made right after a new variety is released. A well-known illustration of this pattern is that approximately $40 \%$ of US cinema box office revenues are obtained during the first week and very few movies generate significant revenue beyond the sixth week. One reason is the heavy advertising campaigns leading up to the release date. A second reason is that consumption externalities play a big role in these markets, and consumers prefer to purchase a particular variety when aggregate consumption hits its highest level. ${ }^{2}$

In this paper we abstract from static product diversity in order to study in a clean and simple environment the market provision of dynamic product diversity. ${ }^{3}$ Thus, the definition of the industry we have in mind involves some degree of disaggregation. As one would expect, looking at product diversity from a dynamic perspective brings about new insights and significantly changes

\footnotetext{
${ }^{1}$ Another workhorse model of product differentiation, the Spence-Dixit-Stiglitz model (Spence, 1976; and Dixit and Stiglitz, 1977), takes a different strategy and describes consumer decisions as emanating from a "representative consumer" with a preference for diversity. But this is only meant as a modeling short-cut rather than as a literal representation of individual consumer behavior.

${ }^{2}$ For a useful discussion of common practices and stylized facts in the motion picture industry, see Corts (2001), Krider and Weinberg (1998), and Einav (2007). On consumption externalities see, for instance, Becker (1991).

${ }^{3}$ As explained below, the choice of ignoring static product differentiation forces us to concentrate on monopoly markets.
} 
the trade-offs identified in the literature.

We present a dynamic model where both the monopolist and consumers are infinitely-lived and form rational expectations. Consumers are ex-ante identical, but have random, variety-specific preferences. Each consumer purchases at most one unit of each variety, and each such purchase affects their willingness to pay for new varieties. We could generally define that preferences are subject to transitory saturation if the utility derived from a new consumption episode tends to increase with the time elapsed since the last episode. Here we adopt a simplified version that contributes significantly to the tractability of the model: a consumer's expected valuation is lower if they did consume the previous variety in the last period. Thus, the preference cycle lasts for only two periods.

The assumption about ex-ante identical consumers with stochastic, varietyspecific preferences is probably a good representation of these real world markets where consumers are likely to disagree on their ranking of their favorite varieties. Moreover, it allows us to avoid Coasian price dynamics, typically studied in the context of durable goods. In fact, when preferences experience transitory saturation, consumption decisions are -in some sense- durable. Thus, in a hypothetical scenario with ex-ante heterogeneous consumers, right after selling the good to consumers with relatively high willingness to pay, a monopolist would face a customer base uniquely composed of individuals with relatively low willingness to pay, since previous buyers would have depreciated valuations for some time.

We assume that the cost of introducing a new variety is independent of the time elapsed since the last variety was introduced. As a result, the frequency of new product introduction is completely demand-driven; that is, it is exclusively dependent on how fast consumers can absorb new varieties, and hence on the intensity of transitory saturation.

We take the degree of product differentiation between two consecutive varieties as exogenously given, and focus exclusively on the speed at which these predetermined varieties are introduced. This is the main reason why we focus on monopoly pricing. A full analysis of interfirm competition would require us to consider not only dynamic product differentiation, but as well differentiation 
at a point in time, and hence an explicit analysis of how product characteristics are sequentially selected by different firms. These important issues are clearly relevant, but beyond the scope of this paper.

The main result of the paper is that the frequency at which a monopolist introduces new varieties may be higher or lower than is socially optimal. Excessive high frequency arises when the impact of transitory saturation is sufficiently strong. Excessive dynamic product variety may coexist with underconsumption. That is, too many varieties are produced but each variety is purchased by too few consumers.

The determination of whether dynamic product variety under monopoly is excessive or insufficient depends on the relative strength of two countervailing effects. These effects are related to those discussed in the literature. In particular, the analysis of horizontal product differentiation in static frameworks has also shown that a monopolist may provide too little or too much product variety with respect to the social welfare maximizing level (See, for instance, the intuitive examples discussed in De Meza and Von Ungern-Stenberg, 1982). There are two effects that work in opposite directions. On the one hand, the monopolist can only appropriate a fraction of total surplus but must pay the full cost of producing a new variety. As a result, the monopolist would tend to underprovide product diversity. On the other hand, product variety allows for a better price discrimination strategy. ${ }^{4}$ Thus, greater product variety implies that the monopolist can appropriate a larger share of total surplus. If the second effect is sufficiently strong then a monopolist provides an excessive degree of product diversity.

In our model the two countervailing effects are somewhat analogous. The appropriability effect is in fact identical to the static model, since in both cases it has to do with the inability of the monopolist to differentiate between different types of consumers. The countervailing effect can be interpreted as a particular form of intertemporal price discrimination. ${ }^{5}$ In our model prices are

\footnotetext{
${ }^{4}$ It is not necessarily the case that more variety implies more price disperssion, in the sense that different consumer types are being charged different prices. What is crucial is that more variety allows the monopolist to discriminate among different consumer groups and charge all of them a higher price.

${ }^{5}$ In most cases, like durable goods, or non-durables under customer recognition, intertem-
} 
constant over time, and hence it is not the case that consumer types pay different prices in different periods. Instead, the monopolist finds it optimal to raise the price above the short-run profit maximizing level in order to allow consumers to recover from previous consumption episodes and thereby increase future sales. Because of such demand cycle, a monopolist is able to appropriate a higher share of surplus as the rate of introduction of new products increases. Hence, as in the static case, whenever the second effect is sufficiently strong then the monopolist is providing too much product variety (i.e., introducing new products too frequently).

The role of transitory saturation in non-durable goods can also be related to the effect of depreciation or quality improvements in durable goods; in the sense that they both induce repeat purchases and generate a negative link between current demand and past purchases. The frequency of innovations can also be discussed if quality improvements are introduced. ${ }^{6}$ There is an extensive literature analyzing product innovation (quality upgrading) in durable goods. In line with the results of our paper, it has been shown that a monopolist may introduce more upgrades than is socially optimal (See, for instance, Waldman, 1993; Choi, 1994; Ellison and Fudenberg, 1998). However, these papers present two-period models and focus on network externalities and compatibility between old and new models, and hence their results are hardly comparable with ours.

Perhaps, the model most closely related to ours is Fishman and Rob (2000), in the sense that they also consider an infinite horizon framework and study how efficient is the frequency of innovations generated under monopoly. A crucial assumption of their model is that innovations are cumulative. They show that a monopolist introduces new products too slowly with respect to the social optimum (at least, in the case of no price discrimination and no planned obsolescence). The reason is that current innovation efforts have a positive

poral price discrimination reduces monopoly profits. In fact, a monopolist would like to commit not to price discriminate. See, for instance, a recent survey by Armstrong (2006). In contrast, in our model intertemporal price discrimination increases the share of the total surplus that a monopolist can appropriate.

${ }^{6}$ The literature on depreciating durable goods has focused on very different issues; for instance, on the role of replacement sales in preventing the Coase conjecture (Bond and Samuelson, 1984; Driskill, 1997), or the effect of scrapping subsidies (Adda and Cooper, 2000). 
effect on all subsequent models, but consumers are only willing to pay for the incremental flow of services the current model provides.

Despite of certain similarities with the case of durable goods, our model focuses on non-durable goods for which consumer preferences are subject to transitory saturation. Below we discuss to what extent the current framework can be adapted to deal with durable goods. We argue durability and transitory saturation have a sufficiently different nature, which is reflected in distinct patterns of consumer behavior.

The plan of the paper is as follows: In the next section we present the baseline model and Section 3 is devoted to the first best allocation. Section 4 contains the main results. In this section we study the game under the assumption that the monopolist can commit up-front to the frequency of new product introductions, but not to prices. It is shown that there exists a unique equilibrium with constant prices. If new varieties are introduced in every period, then both the monopolist and consumers engage in speculative behavior. On the one hand, consumers become choosier as the option value of waiting is positive: they anticipate that their current consumption would dampen the expected valuation of the variety that will be supplied in the next period. On the other hand, the monopolist finds it optimal to set a price above the level that maximizes shortrun profits. Alternatively, if new varieties are introduced in every other period, then all intertemporal effects vanish and optimal decisions are static. It turns out that in the first scenario the monopolist is able to appropriate a larger share of the total surplus, which explains why -in some region of parameter valuesthe frequency of new product introductions is socially excessive. In Section 5 we study the game in case the monopolist cannot precommit to the frequency of new product introductions. It is shown that the lack of commitment generates multiple equilibria and, moreover, the region of parameter values with excessive product variety is expanded. Finally, in Section 6 we discuss the differences between transitory saturation and durability, the monopoly game under price commitment, and the impact of competition in a restricted environment. 


\section{The model}

We examine an infinite horizon model of a non-durable good industry in which consumer preferences are subject to transitory saturation. We let $t$ index periods, $t=0,1,2, \ldots$ We consider a single, infinitely-lived producer who sequentially supplies different varieties of the good. In each period the monopolist can introduce a new variety by incurring a fixed cost, $F$. Any amount of the variety can then be produced at constant marginal cost, which is normalized to 0. Every time a new variety is introduced the previous variety ceases to be available. The monopolist chooses both the frequency of introduction of new varieties and their production level in order to maximize the expected discounted value of profits, using the discount factor, $\delta \in[0,1)$.

There is a mass one of infinitely-lived consumers with history-dependent preferences. Each consumer purchases at most one unit of each variety. Consumer preferences are variety-specific and depend on past consumption. More specifically, if consumer $i$ did not consume a previous variety in period $t-1$, then her valuation of the new variety in period $t, r_{i t}$, is a random variable, uniformly distributed on the interval $[0,1]$ (distribution $N C$ ). Moreover, her valuation in subsequent periods remains constant, either until she purchases the first unit of the variety or a new variety is introduced. However, if consumer $i$ did consume in period $t-1$, then $r_{i t}$ is a realization of distribution $C$, such that:

$$
r_{i t}=\left\{\begin{array}{c}
0, \text { with probability } 1-\mu \\
\sim U[0,1], \text { with probability } \mu
\end{array}\right.
$$

where $\mu \in[0,1)$ is a fixed parameter. If $r_{i t}>0$ then this is also her valuation of (the first unit of) the variety in subsequent periods. If $r_{i t}=0$, then in period $t+1$ she obtains a realization from distribution $N C$, which again remains constant, until a new variety is introduced.

Thus, conditional on not having consumed in period $t-1$, a consumer's expected valuation of a new variety in period $t$ is $\frac{1}{2}$. However, if she did consume in the previous period, her expected valuation is $\frac{\mu}{2}<\frac{1}{2}$. Thus, a lower value of parameter $\mu$ indicates a stronger (negative) effect of past consumption on current preferences. Note that -for the purpose of tractability- the preference cycle lasts for two periods. Also, consumers are ex-ante identical but heterogenous 
ex-post. In other words, there is a representative consumer, but given that decisions are taken after observing their current valuations, at any point in time the monopolist faces a smooth, downward sloping demand function.

We denote by $\alpha_{t}$ the fraction of consumers that in period $t$ obtain a positive realization of $r_{i t}$. Clearly, $\alpha_{t}$ will depend on $\alpha_{t-1}$ and the consumption behavior in period $t-1$, in a way that will be specified below. Thus, $\alpha_{0}$ is one of the exogenous parameters of the model, but $\alpha_{t}$, for all $t>0$, are endogenous variables. For simplicity, we restrict attention to the case of $\alpha_{0}=1$. This is equivalent to focusing on the medium and long-run performance of the industry. Considering an arbitrary value of $\alpha_{0} \in[0,1]$ significantly complicates the analysis and brings about little additional insights.

An individual obtains a net surplus of $r_{i t}-p_{t}$ if she chooses to consume in period $t$ at a price $p_{t}$. Otherwise she gets 0 . Consumers are infinitely-lived and maximize the expected discounted value of their net surplus, using the same discount factor, $\delta$.

For future reference we let $z \equiv \delta(1-\mu)$, which indicates the intensity of transitory saturation. Low values of $\mu$ will have a substantial effect on current behavior only if consumers are forward looking ( $\delta$ is high).

Thus, the model aims at understanding how the frequency of introduction of new varieties is determined in markets in which consumers typically purchase a single unit of each variety, but the recent history of purchases affect the valuation of new varieties (think, for instance, of films and books). ${ }^{7}$ Since consumer preferences are variety-specific, commercialization and consumption occur simultaneously. Also, note that the model is sufficiently abstract so that the characteristics of subsequent varieties (and therefore the "distance" between them) is not made explicit. Consequently, alternative interpretations are also possible. For example, the model can also capture the case of highly perishable goods (like concerts) that are available exclusively at particular points in time. Whether or not the goods offered at different points in time are horizontally differentiated is not important in this interpretation. Finally, those markets where

\footnotetext{
${ }^{7}$ In the case of music recordings and computer games consumers typically make a repeated use of each variety. To the extent that most of these consumption episodes occur right after the acquisition of the good, then the model can be though of as fitting these markets as well.
} 
essentially the same variety is continuously available but consumers experience transitory saturation (amusement parks, restaurants, tourist destinations) also fit with our model. Obviously, in this case we cannot discuss the frequency of introduction of new products.

\section{The first best}

An allocation can be described as a pair $\left(\gamma_{t}, I_{t}\right)$ for each period $t=0,1,2, .$. , where $\gamma_{t}=1$ indicates that a new variety has been introduced in period $t$ (and the fixed cost is paid), while $\gamma_{t}=0$ indicates no new variety. $I_{t} \subseteq[0,1]$ represents the set of values of $r_{i}$ for which individuals consume in a given period. In this section we characterize the allocation that maximizes the expected discounted value of total surplus (profits plus consumer surplus). We start by examining the optimal consumption pattern conditional on $\gamma_{t}=1$ for all $t$. Next, we consider alternative frequencies of new product introductions and their associated consumption patterns.

Suppose a new variety is introduced every period. Since marginal costs are constant, the optimal choice of an individual consumer only depends on her own realization, $r_{i t}$, and not on the aggregate consumption level. If consumer $i$ acquires the good then her expected level of utility is $r_{i t}+\delta U_{t+1}^{C}$, where $U_{t+1}^{C}$ is the continuation value at the beginning of period $t+1$, before she gets a draw

from distribution $C$. If she waits (does not consume) then she gets $\delta U_{t+1}^{N C}$, where $U_{t+1}^{N C}$ is the continuation value at the beginning of period $t+1$, before she gets a draw from distribution $N C$. Note that neither $U_{t+1}^{C}$ nor $U_{t+1}^{N C}$ depend on $r_{i t}$. Thus, from an efficiency point of view, consumer $i$ should consume if and only if $r_{i t} \geq \bar{r}_{t}$, where

$$
\bar{r}_{t}=\delta\left(U_{t+1}^{N C}-U_{t+1}^{C}\right)
$$

In other words, without loss of generality, we can write $I_{t}=\left[\bar{r}_{t}, 1\right]$. Thus, an efficient allocation can be described by a sequence of threshold values, $\left\{\bar{r}_{t}\right\}_{t=0}^{\infty}$, that maximizes the present value of total surplus:

$$
W_{0}=\sum_{t=0}^{\infty} \delta^{t} \alpha_{t} T S\left(\bar{r}_{t}\right)
$$


where $T S\left(\bar{r}_{t}\right)=\int_{\bar{r}_{t}}^{1} r d r$ is the expected total surplus per consumer generated in period $t$, given the threshold $\bar{r}_{t}$. Note that $\alpha_{t}$ is determined by past consumption behavior. More specifically, aggregate consumption in period $t-1$ was $\left(1-\bar{r}_{t-1}\right) \alpha_{t-1}$. A fraction $\mu$ of these consumers, plus all those who did not consume, $1-\left(1-\bar{r}_{t-1}\right) \alpha_{t-1}$, will be able to draw in period $t$ a positive $r_{i t}$. Therefore, the law of motion of $\alpha_{t}$ is:

$$
\alpha_{t}=1-(1-\mu)\left(1-\bar{r}_{t-1}\right) \alpha_{t-1}
$$

The solution of this optimization problem is characterized in the following lemma:

Lemma 1 If a new variety is introduced every period, it is efficient that consumers purchase the good if and only if $r_{i t} \geq \bar{r}^{*}$, where:

$$
\bar{r}^{*}=\frac{1+z-\sqrt{1+2 z}}{z}
$$

In the Appendix we prove the Lemma.

Note that $\bar{r}^{*}$ is an increasing function of $z$, with $\bar{r}^{*}(0)=0$, and $\bar{r}^{*}(1)=2-$ $\sqrt{3}$. As $z$ increases, consumers become more selective because the opportunity cost of waiting, $\delta\left(U^{N C}-U^{C}\right)$, also increases.

Since the optimal threshold value is constant over time, the law of motion becomes:

$$
\alpha_{t}=1-(1-\mu)\left(1-\bar{r}^{*}\right) \alpha_{t-1} .
$$

Since $0<(1-\mu)\left(1-\bar{r}^{*}\right)<1$, then $\alpha_{t}$ converges to the steady state value $\alpha^{*}=\frac{1}{1+(1-\mu)\left(1-\bar{r}^{*}\right)}$, following an oscillating trajectory.

Let us now turn to the endogenous determination of the frequency of new product introduction. All possible optimal trajectories can be identified with the number of consecutive innovative periods (in which a new variety is introduced), $N$, that precede an idle period (no new variety). In other words, for an arbitrary $N, \gamma_{0}=\gamma_{1}=\ldots=\gamma_{N-1}=1$ and $\gamma_{N}=0$. As a result, $\alpha_{N+1}=1$ and a new cycle of $N$ consecutive innovative periods commences. In particular, one of the possible optimal paths consists of introducing a new variety every period $(N=\infty)$, which can be called option $(\infty)$; its associated payoff can be computed by rewriting equation (2) using equation (4): 


$$
W^{\infty}=\frac{\alpha_{0}+\frac{\delta}{1-\delta}}{A^{*}}-\frac{F}{1-\delta}=\frac{1}{1-\delta}\left(\frac{1}{A^{*}}-F\right)
$$

where $A^{*}=\frac{1+z\left(1-\bar{r}^{*}\right)}{R\left(\bar{r}^{*}\right)}$. Note that $A^{*}$ increases with $z$.

A second possible trajectory consists of introducing a new variety every other period $(N=1)$, starting with period 0 , i.e., $\gamma_{t}=1$ if and only if $t=0,2,4, \ldots \mathrm{In}$ this case, in all subsequent innovative periods $\alpha_{t}=1$, independently of current consumption decisions. The value of waiting is 0 and consequently it is efficient to let agents consume, provided $r_{i} \geq 0$. The expected payoff of option (1) is:

$$
W^{1}=\alpha_{0} R(0)-F+\frac{\delta^{2}}{1-\delta^{2}}(R(0)-F)=\frac{1-2 F}{2\left(1-\delta^{2}\right)}
$$

In the Appendix we prove the following Lemma.

Lemma 2 If $F<\frac{1}{2}$, in a first best allocation either a new variety is introduced every period $(N=\infty)$ or every other period $(N=1)$.

Thus, the first best can be determined by simply comparing equations (5) and (6). If we let $F^{*}=\frac{1}{\delta}\left(\frac{1+\delta}{A^{*}}-\frac{1}{2}\right)$ then:

Proposition 3 The first best allocation consists of: (i) Introducing a new variety every period $\left(\gamma_{t}=1\right.$ for all $\left.t \geq 0\right)$ and letting all agents with $r_{i t} \geq \bar{r}^{*}$ consume, if $F \in\left[0, F^{*}\right]$, (ii) Introducing a new variety every other period $\left(\gamma_{t}=1\right.$ if and only if $t=0,2,4, \ldots)$ and letting all agents with $r_{i t} \geq 0$ consume, if $F \in\left[F^{*}, \frac{1}{2}\right]$.

\section{The frequency of new product introductions under commitment}

This is a model where expectations matter and, as a result, the monopoly solution is time inconsistent. Moreover, there may exist multiple equilibria. In this section we examine the case where the monopolist can choose up-front the frequency of new product introduction, but the prices of future varieties cannot be predetermined. In the next section, we further restrict the ability of the monopolist to commit to future production plans. In Section 6 we comment on, among other things, monopoly pricing under full commitment. 
At time 0 we let the monopolist select the entire production plan $\gamma_{t} \in\{0,1\}$. In those periods in which a new variety is introduced, the firm sets the price of the variety, $p_{t}$, and it remains valid until the next variety is introduced. After observing the availability of a new variety, its price, $p_{t}$, and their own realizations, $r_{i t}$, consumers decide whether or not to consume. We restrict attention to Markov strategies; that is, monopoly price depends exclusively on $\alpha_{t}$, and consumer decisions depend on $\alpha_{t}$ and $p_{t}$. Moreover, we focus on perfect Markov equilibria where prices (and consumer behavior) are constant over time. That is, along the equilibrium path, $p_{t}=p$, and hence $\bar{r}_{t}=\bar{r}$. Note that in this type of equilibrium consumers do not have any incentives to delay the purchase of a particular variety since preferences are variety-specific and prices are constant. Hence, commercialization and consumption are perfectly synchronized.

It will be useful to start analyzing the case in which a new variety is introduced every period.

\subsection{Preliminaries: a new variety is introduced every pe- riod}

Suppose $\gamma_{t}=1$ for all $t$. In this case, the strategies of the firm and consumers can be written as $p\left(\alpha_{t}\right)=p^{m}$ and $\bar{r}\left(p_{t}, \alpha_{t}\right)=f\left(p_{t}\right)$, respectively. We will denote by $\bar{r}^{m}$ the threshold value along the equilibrium path, i.e., , $\bar{r}^{m}=f\left(p^{m}\right)$. In equilibrium, $p^{m}$ is the price that maximizes the expected value of profits, under the beliefs that consumers behave according to $\bar{r}\left(p_{t}, \alpha_{t}\right)=f\left(p_{t}\right)$ and that $\left(p^{m}, \bar{r}^{m}\right)$ will prevail in the future, and $\bar{r}\left(p_{t}, \alpha_{t}\right)=f\left(p_{t}\right)$ describes consumers' optimal behavior under the beliefs that $\left(p^{m}, \bar{r}^{m}\right)$ will prevail in the future.

\section{Consumer decisions}

If a consumer with $r_{i t}$ purchases the good then her payoff is $r_{i t}-p_{t}+\delta U_{t+1}^{C}$, otherwise she obtains $\delta U_{t+1}^{N C}$. Along a constant price equilibrium, $U_{t+1}^{N C}$ and $U_{t+1}^{C}$ are independent of $p_{t}$ and constant over time. Thus, for all $t$ :

$$
\bar{r}_{t}=f\left(p_{t}\right)=p_{t}+\delta\left(U^{N C}-U^{C}\right)
$$


Note that $\frac{\partial \bar{r}_{t}}{\partial p_{t}}=1$. Consumers compare the instantaneous utility from consumption, $\bar{r}_{t}-p_{t}$, with the option value of waiting, $\delta\left(U^{N C}-U^{C}\right)$, and hence purchase if and only if $r_{i t}$ is sufficiently higher than $p_{t}$. In fact, along a constant price equilibrium we have that:

$$
\begin{gathered}
U^{N C}=C S(\bar{r}, p)+(1-\bar{r}) \delta U^{C}+\bar{r} \delta U^{N C} \\
U^{C}=\mu C S(\bar{r}, p)+\mu(1-\bar{r}) \delta U^{C}+(\mu \bar{r}+1-\mu) \delta U^{N C}
\end{gathered}
$$

where $C S(\bar{r}, p) \equiv \int_{\bar{r}}^{1}(r-p) d r$ is the per period expected consumer surplus for an individual that did not consume in the previous period. If we solve these two equations for $U^{N C}-U^{C}$, and substitute this value in equation (7) then we have:

$$
\bar{r}_{t}=f\left(p_{t}\right)=p_{t}+\frac{z C S(\bar{r}, p)}{1+z(1-\bar{r})}
$$

If we evaluate this expression at $\bar{r}_{t}=\bar{r}$, and $p_{t}=p$, we derive one of the two equations that characterize a constant price equilibrium:

$$
p=\bar{r}[1+z(1-\bar{r})]-\frac{z}{2}\left(1-\bar{r}^{2}\right)
$$

Note that $\bar{r}$ increases with $p$, and if $p=0$ then $\bar{r}=\bar{r}^{*}$. Unsurprisingly, if the price is above marginal cost, then there is underconsumption.

\section{Firm's optimal pricing}

In period $t$ the monopolist's payoff is:

$$
\Pi\left(\alpha_{t}\right)=\arg \max _{p_{t}} \alpha_{t}\left[1-f\left(p_{t}\right)\right] p_{t}-F+\delta \Pi\left(\alpha_{t+1}\right)
$$

where $f\left(p_{t}\right)$ is given by equation $(9), \Pi\left(\alpha_{t+1}\right)$ is the continuation value at the beginning of period $t+1$, and $\alpha_{t+1}$ is:

$$
\alpha_{t+1}=1-(1-\mu)\left(1-\bar{r}_{t}\right) \alpha_{t}
$$

Note that $\frac{\partial \alpha_{t+1}}{\partial \bar{r}_{t}}=(1-\mu) \alpha_{t}$, and $\frac{\partial \alpha_{t+1}}{\partial \alpha_{t}}=-(1-\mu)(1-\bar{r})$.

In a constant price equilibrium $\Pi(\alpha)$ must be a linear function of $\alpha$; i.e., $\frac{d \Pi\left(\alpha_{t+1}\right)}{d \alpha_{t+1}} \equiv k$, which is independent of $\alpha_{t}$. Then, the first order condition of the firm's optimization problem (the second order condition is satisfied) is:

$$
\left(1-\bar{r}_{t}-p_{t}\right)+k z=0
$$


The first important observation to make is that the optimal $p_{t}$ does not depend on $\alpha_{t}$, which is consistent with the existence of a constant price equilibrium. The second observation is that transitory saturation induces the firm to set a price above the level that maximizes current profits. In other words, if the firm increases its price slightly above the level that maximizes static profits, it causes a second order loss on current profits but it raises future profits (first order effect) by increasing demand in the next period (provided $k>0$ ).

By the envelop theorem, if we evaluate $\Pi\left(\alpha_{t}\right)$ at the constant price equilibrium then:

$$
k=\frac{d \Pi\left(\alpha_{t}\right)}{d \alpha_{t}}=(1-\bar{r}) p-k z(1-\bar{r})
$$

Hence, $k$ is independent of $\alpha_{t}$. Solving for $k$ and substituting the value in equation (10) we obtain:

$$
p_{t}=\left(1-\bar{r}_{t}\right)+\frac{z(1-\bar{r}) p}{1+z(1-\bar{r})}
$$

If we evaluate this expression at $\bar{r}_{t}=\bar{r}$, and $p_{t}=p$, we derive the second equation that determines a constant price equilibrium:

$$
p=(1-\bar{r})[1+z(1-\bar{r})]
$$

\section{Equilibrium}

Equations (9) and (12) uniquely determine the equilibrium values of $\left(p^{m}, \bar{r}^{m}\right)$ :

$$
\begin{aligned}
\bar{r}^{m} & =\frac{2+3 z-\sqrt{4+6 z}}{3 z} \\
p^{m} & =\frac{2+6 z-\sqrt{4+6 z}}{9 z}
\end{aligned}
$$

The red lines in Figure 1 depict, on the one hand, the locus describing optimal consumer choices (equation 9) and, on the other, the optimal monopoly prices (equation 12), in the case $z=0$. Similarly, the blue lines correspond to the case $z=1$. Note that $\bar{r}^{m}$ is an increasing function of $z$, with $\bar{r}^{m}(z=0)=\frac{1}{2}$, and $\bar{r}^{m}(z=1)=\frac{5-\sqrt{10}}{3}$. Graphically, the effect of $z$ on $\bar{r}^{m}$ is the result represented by both blue lines shifting towards the right and thus reinforcing each other. However, the effect of $z$ on $p^{m}$ is apparently ambiguous since it is the net result of two countervailing effects: consumer behavior tends to reduce prices 
(demand function shifts downwards due to consumers' speculative behavior, which reduces the price that maximizes current profits), but the monopolist finds it optimal to raise the price above the level that maximizes current profits. Algebraically, we can see that $p^{m}$ is also an increasing function of $z$, with $p^{m}(z=0)=\frac{1}{2}$ and $p^{m}(z=1)=\frac{8-\sqrt{10}}{9}$. Finally, $\bar{r}^{m}(z)>\bar{r}^{*}(z)$.

We can further analyze the effect of $z$ on $p^{m}$ by examining equations (8) and (11). The departure from the static optimal consumer behavior, $\frac{z C S(\bar{r}, p)}{1+z(1-\bar{r})}$, is a proportion of static consumer surplus. Similarly, the departure from the static monopoly price, $\frac{z(1-\bar{r}) p}{1+z(1-\bar{r})}$, is the same proportion of static profits. In fact, combining these two equations we can write:

$$
p=\frac{1}{2}\left\{1+\frac{z}{1+z(1-\bar{r})}[(1-\bar{r}) p-C S(\bar{r}, p)]\right\}
$$

Since monopoly profits are higher than consumer surplus, prices are above the equilibrium level of the static game. Thus, on the one hand, we can anticipate than in an oligopoly model (with lower equilibrium prices and hence higher consumer surplus and lower profits) the net effect is likely to have the opposite sign, and equilibrium prices may turn out to be lower than in the static game. However, on the other hand, the presence of myopic consumers would reduce the impact of transitory saturation on consumer behavior and reinforce the positive effect on equilibrium prices.

Summarizing, if production takes place every period, $N=\infty$, then equilibrium prices and consumer behavior $\left(p^{m}, \bar{r}^{m}\right)$ are given by equations (13) and (14). Hence, the present value of profits can be written as:

$$
\Pi^{\infty}\left(\alpha_{0}\right)=\frac{\alpha_{0}+\frac{\delta}{1-\delta}}{A^{m}}-\frac{F}{1-\delta}=\frac{1}{1-\delta}\left(\frac{1}{A^{m}}-F\right)
$$

where $A^{m}=\frac{1+z\left(1-\bar{r}^{m}\right)}{\left(1-\bar{r}^{m}\right) p^{m}}$.

We can now compare monopoly profits with potential surplus (gross of fixed costs). This ratio is equal to $\frac{A^{*}}{A^{m}}$. The analysis of this ratio provides us with the following result:

Remark 4 The ratio of profits to potential surplus is increasing in $z$ and takes values in the interval $[0.5,0.56]$. 


\subsection{The optimal monopoly plan}

Let us now consider alternative patterns of new product introductions. Using the labels introduced in Section 3, option (1) consists of introducing new varieties in periods $t=0,2,4, .$. In this case, both the firm's and consumers' optimization problems become static. Consumers anticipate that in the next innovative period they will draw their $r_{i}$ from distribution $N C$ independently of their current behavior; hence they purchase the good if and only if $r_{i t} \geq p_{t}$. Similarly, the monopolist anticipates that demand in the next production period is independent of current prices; hence she sets the current price in order to maximize static profits. As a result, $\bar{r}_{t}=p_{t}=\frac{1}{2}$ (profits per production period are equal to $\alpha_{t} \frac{1}{4}-F$ ) and the firm's payoff is given by:

$$
\Pi^{1}\left(\alpha_{0}\right)=\alpha_{0} \frac{1}{4}-F+\frac{\delta^{2}}{1-\delta^{2}}\left(\frac{1}{4}-F\right)=\frac{1}{1-\delta^{2}}\left(\frac{1}{4}-F\right)
$$

The following Lemma indicates that there is no need to consider any other production plan (the proof can be found in the Appendix):

Lemma 5 If $F<\frac{1}{4}$, then the monopolist finds it optimal to introduce new products every period $(N=\infty)$ or every other period $(N=1)$.

Therefore, the optimal frequency of new product introductions can be characterized by comparing equations (15) and (16). If we let $F^{m}=\frac{1}{\delta}\left(\frac{1+\delta}{A^{m}}-\frac{1}{4}\right)$ we can state the following result:

Proposition 6 If the firm can choose in period 0 the frequency of introduction of new products, there is a generically unique constant price equilibria, that consists of: (i) new varieties are introduced every period, and sold at a price $p^{m}$ to consumers with $r_{i} \geq \bar{r}^{m}$, if $F \in\left[0, F^{m}\right]$, and (ii) new varieties are introduced every other period, sold at a price $\frac{1}{2}$ to consumers with $r_{i} \geq \frac{1}{2}$, if $F \in\left[F^{m}, \frac{1}{4}\right]$.

In order to asses the efficiency of the frequency of new product introduction chosen by a monopolist, we simply need to compare $F^{*}$ and $F^{m}$. Suppose $\delta$ is close to zero. In this case, $F^{*}$ is close to $\frac{1}{2}$ and $F^{m}$ is close to $\frac{1}{4}$. Therefore, $F^{*}>F^{m}$. In this case, monopoly power tends to slow down the rate of new product introduction with respect to the first best. However, if $\mu=0$, as $\delta$ 
goes to 1 , then $F^{*}$ goes to 0.0360 and $F^{m}$ goes to 0.0502 . That is, if transitory saturation is sufficiently strong ( $z$ sufficiently close to 1 ), then $F^{*}<F^{m}$. In this case, a monopolist tends to introduce new products too often with respect to the first best. Summarizing:

Remark 7 Under commitment, the frequency of introduction of new varieties can be higher or lower than in the first best. In particular, new varieties are introduced too frequently when transitory saturation is sufficiently strong.

Monopoly power causes two types of inefficiency. First, the static price distortion leads to underconsumption whenever a new variety is available. Second, the frequency of new product introduction is typically inefficient, although its sign is ambiguous. To explain this ambiguity it is helpful to consider an scenario where the monopolist is able to appropriate a fraction $\lambda$ of potential surplus under all circumstances. With respect to the first best, the payoff differential between the two alternative frequencies (gross of fixed costs) is scaled down by $\lambda$, but nevertheless it has to incur the entire fixed cost. In this case, the firm's limited ability to appropriate surplus tends to slow down the frequency of production. However, under transitory saturation the fraction of the surplus appropriated by the monopolist varies with the frequency of production. If a new variety is introduced every other period (and $\bar{r}=p=\frac{1}{2}$ ) then the monopolist captures one half of potential surplus (gross of fixed costs). However, if a new product is introduced every period then the fraction of potential surplus captured by the monopolist exceeds one half. ${ }^{8}$ The reason is that a forwardlooking monopolist can make higher profits by raising a new product's price above the short-run profit maximizing level, letting consumers' valuations for it grow and capture the surplus in the next period at a higher price. It is true that consumers also become more selective and only purchase the good if the current surplus is sufficiently high. However, this form of consumer speculation also takes place in the first best. Moreover, the monopolist sets a price above marginal costs, which reduces consumers' value of waiting (reduces $U^{N C}-U^{C}$ ), as well as the gap, $\bar{r}-p$. As consumers become less defensive the firm can

\footnotetext{
${ }^{8}$ See Remark 4.
} 
capture a higher fraction of total surplus. Thus, if the impact of transitory saturation is sufficiently strong, the monopolist finds it optimal to introduce new varieties faster than in the first best, even though it sells them to too few consumers. In other words, excessive product variety may actually coexist with underconsumption.

\section{The frequency of new product introductions without commitment}

It was convenient in the prior section to assume the monopolist can choose in period 0 the frequency of new product introductions for the entire game. This assumption about the firm's commitment power may be unrealistic, and this section will examine the consequences of relaxing it. In the absence of commitment, in period $t$ the firm chooses $\gamma_{t}$, and if $\gamma_{t}=1$ then also sets the price of the new variety, $p_{t}$. Consumer behavior is qualitatively the same, although in this case consumers must form expectations about the future pattern of new product introductions. The central role of expectations opens the door to multiplicity of equilibria, at least for some key parameter values. Also, it turns out that without commitment the set of values of the fixed cost for which the equilibrium frequency of new product introduction is socially excessive is larger than in the case of commitment. To illustrate these claims we focus on the particular case of $\mu=0$ and $\delta=1$, i.e., $z=1$.

Type I equilibrium

Consider a particular strategy profile that supports an introduction of a new product in every period. The firm's strategy is:

$$
\left\{\begin{array}{c}
\text { If } \alpha_{t} \in[\bar{\alpha}, 1], \gamma_{t}\left(\alpha_{t}\right)=1, p_{t}\left(\alpha_{t}\right)=p^{m} \\
\text { If } \alpha_{t} \in[0, \bar{\alpha}), \gamma_{t}=0
\end{array}\right.
$$

Let us define by $\bar{p}\left(\alpha_{t}\right)$ the price such that, given the optimal consumer reaction, it induces $\alpha_{t+1}=\bar{\alpha}$. That is, for any $p_{t} \geq \bar{p}\left(\alpha_{t}\right)$ then it is rational to expect that $\alpha_{t+1} \geq \bar{\alpha}$. Thus, consumers' optimal response to the firm's strategy is given by:

$$
\bar{r}\left(p_{t}, \alpha_{t}\right)=\left\{\begin{array}{c}
p_{t}+\Delta, \text { if } p_{t} \geq \bar{p}\left(\alpha_{t}\right) \\
p_{t}, \text { otherwise }
\end{array}\right.
$$


where $\bar{p}\left(\alpha_{t}\right)=1-\Delta-\frac{1-\bar{\alpha}}{\alpha_{t}}$, and $\Delta \equiv \bar{r}^{m}-p^{m}=\frac{4+3 z-\sqrt{4+6 z}}{9 z}=0.07505$. Such strategy profiles will form an equilibrium under some parameter values (See Appendix for details):

- Result 1: A type I equilibrium exists if and only if $F \leq 0.0669$.

Type II equilibrium

Consider now a strategy profile that supports an introduction of a new product every other period. The firm's strategy is:

$$
\left\{\begin{array}{c}
\text { If } \alpha_{t} \in[\bar{\alpha}, 1], \gamma_{t}\left(\alpha_{t}\right)=1, p_{t}\left(\alpha_{t}\right)=\frac{1}{2} \\
\text { If } \alpha_{t} \in(\underline{\alpha}, \bar{\alpha}), \gamma_{t}\left(\alpha_{t}\right)=1, p_{t}\left(\alpha_{t}\right)=\frac{5}{8}
\end{array}\right.
$$

Given such a strategy profile, optimal consumer behavior for all possible prices and values of $\alpha_{t}$ is more complicated that in the case of type I equilibria. Further details and the analysis of possible deviations are given in the Appendix, where it is shown that: ${ }^{9}$

- Result 2: A type II equilibrium exists if and only if $F \in(0.0562,0.0750)$.

Remark 8 If $F \in(0.0562,0.0669)$ both type I and type II equilibria exist.

Let us provide some intuition about the multiplicity of equilibria. Suppose $\alpha_{t}=1$. If both the firm and consumers expect that new products will be introduced every period, then consumers find it optimal to purchase only if their current net surplus is sufficiently high (higher than $\Delta$ ), and firms prefer to set a relatively high price $\left(p^{m}>\frac{1}{2}\right)$. As a result, sales are relatively low, and $\alpha_{t+1}$ turns out to be relatively high. Consequently, the firm finds it optimal to introduce a new variety in period $t+1$, given the high level of demand. In contrast, if consumers and the firm expect that $\gamma_{t+1}=0$, then consumers purchase the new variety even if the current surplus is arbitrarily low, but positive, and the firm prefers to set a relatively low price $\left(\frac{1}{2}\right)$. As a result, sales are abundant and $\alpha_{t+1}$ turns out to be low. Consequently, the firm prefers not to introduce a new variety in period $t+1$, given the relatively low state of demand.

\footnotetext{
${ }^{9}$ If $F>0.0651$ there exists another type of equilibrium that supports an introduction of a new product every other period.
} 
When both types of equilibrium exist it may be interesting to learn which is preferred. In the case $\mu=0$ and $\delta=1$ the payoffs per period for each type of equilibrium are given in the following table.

$\begin{array}{ccc} & \text { Type I equilibrium } & \text { Type II equilibrium } \\ \text { Profits } & 0.1501-F & 0.125-\frac{F}{2} \\ \text { Consumer surplus } & 0.0750 & 0.0625 \\ \text { Total surplus } & 0.2251-F & 0.1875-\frac{F}{2}\end{array}$

Thus, consumers always prefer the type I equilibrium in spite of higher prices, since the good is continuously available. In contrast, the firm prefers the type I equilibrium if and only if $F \leq 0.0502$. Finally, a utilitarian social planner always prefers the type I equilibrium. Thus, when both types of equilibrium exist there is a conflict of interest, and consumers and the firm disagree about which equilibrium they should play. Also, a second best policy that can only dictate the frequency of new product introduction, but cannot avoid monopoly pricing, would only intervene to break an equilibrium where new products are introduced every other period. In other words, from a second best point of view new products may be introduced too slowly, but never too quickly.

\section{Discussion}

\subsection{Transitory saturation and durability}

As discussed in the introduction, the pattern of repeat purchases of non-durable goods subject to transitory saturation exhibits some common features with the case of durable goods subject to either depreciation or quality improvements. In all these cases, a current customer is less likely to purchase again in the near future. We need now to address the question: To what extent can the above model also be interpreted as a model of durable goods.

First of all, some features of the model, especially the assumption that consumer preferences are variety-specific, may be a good approximation in markets for most leisure goods, but may be less so in the case of most durable goods. 
Even if we keep the random structure of individual consumer preferences unchallenged, there are important differences in the determinants of consumer behavior depending on whether the good is durable or non-durable, but subject to transitory saturation.

Let's first focus on the case of durable goods that depreciate over time, but where no quality improvements are introduced. By definition we cannot discuss the frequency of new product introductions, but we can still discuss other issues -like the endogenous determination of durability. Suppose that in each period the utility that consumer $i$ obtains from the use of the good, $r_{i t}$, is an i.i.d. random variable uniformly distributed over the interval $[0,1]$. A good lasts a second period with probability $1-\mu \in[0,1]$. Thus, $1-\mu$ is also an index of durability. Let us now examine consumers' decisions. If we let $U^{*}$ be the continuation value in case the consumer does not own a working unit, then it is optimal to purchase the good if and only if:

$$
r_{i t}-p_{t}+\delta\left[(1-\mu)\left(\frac{1}{2}+\delta U^{*}\right)+\mu U^{*}\right] \geq \delta U^{*}
$$

That is, if and only if $r_{i t} \geq \bar{r}_{t}$, which is:

$$
\bar{r}_{t}=p_{t}-\delta(1-\mu) \frac{1}{2}+(1-\mu)(1-\delta) \delta U^{*}
$$

Thus, as in the case of non-durable goods subject to transitory saturation, consumers purchase the good only if the "short-run" surplus, $r_{i t}+\delta(1-\mu) \frac{1}{2}-p_{t}$, is above a certain threshold, $(1-\mu)(1-\delta) \delta U^{*}$. However, it is important to note that such a threshold goes to 0 as $\delta$ approaches 1 . In other words, if the short-run surplus is arbitrarily small, then consumers are not willing to purchase the good simply because it would delay (in expected terms) the gains derived from future purchases. Hence, this is sufficiently different from the above model (equation (7)) where the value of waiting is the difference between two alternative continuation values, and such difference does not vanish (just the opposite, it grows) as consumers become infinitely patient.

Consider now the case of durable goods that do not depreciate, but where suppliers can introduce quality improvements. In this case, consumers' random preferences imply that at a point in time there are different consumers groups, and consumers in each own a unit of a different age. Thus, the dimension of 
the vector of state variables grows over time, and the tractability of the model comes into question.

In any case, the analogy between durable goods and non-durables subject to transitory saturation is worth exploring in future work.

\subsection{Price commitment}

In case where the monopolist introduces new products every other period, then future prices play no role in current consumers' decisions. As a result, the ability to commit to future prices is worthless. However, when new products are introduced every period, then commitment power ceases to be redundant. In fact, with respect to the time consistent equilibrium characterized above, the firm would like to commit to higher future prices. The reason is that a deviation in future prices around the optimal level has a second order effect on future profits, but as well has a first order effect on current profits, since the expectation of higher future prices reduces consumers' value of waiting, which in turn increases current demand.

As a result, the ability of committing to future prices enhances the monopolist's capacity to appropriate surplus, and this expands the range of parameter values under which the frequency of new product introductions is socially excessive (with respect to the first best).

\subsection{Competition}

As mentioned in the introduction a full-fledged analysis of competition requires an explicit consideration of the characteristics of all the products supplied at different points in time. Such a demanding task is left for future research. In some sense, the goal would be to embed the substitutability between consumption in different periods outlined in this paper with the static substitutability of standard models of product differentiation.

Here we can offer some hints of the effects of competition in the extreme case that static substitutability becomes arbitrary large. In other words, sup-

pose that we allow for more than one firm in our framework, but all firms are constrained to produce the same variety in a given period of time. In other 
words, if more than one firm decides to enter the market in a given period (and pay the fixed cost) then they would be offering perfect substitutes, which would drive prices down to marginal cost. Hence, all active firms would be making negative profits in that particular period. In this scenario, interfirm competition would essentially become a game of timing.

It is possible to read off from this context the following results. First, in the absence of implicit or explicit coordination, firms should be expected to take production decisions simultaneously and play mixed strategies. In other words, in the unique symmetric Markov equilibrium, each firm enters the market in a given period with a probability lower than one. As a result, the number of active firms in any period is a random variable. Actually, the lack of coordination is likely to hurt consumers since, despite the fiercer price competition, in some periods no new variety will be introduced.

Second, in this context it is natural to think of the existence of some coordinating device. In order to fix ideas, consider a duopoly and suppose that the two firms have implicitly agreed on the timing of production, with one of the firms producing in even periods and the other in odd periods. Such an agreement is self-enforcing for a large set of parameter values. In this case, each active firm is in effect a transitory monopolist, but the existence of the rival firm will affect prices through the intertemporal demand effect. Let us examine the existence of equilibria in which a new variety is introduced every period and prices are constant over time.

If consumers expect that a new variety will be available every period, then their behavior is fully characterized by equation (9). In short, $\bar{r}^{d}=p+\Delta^{d}$. Let's now look closely at the problem of the firm that introduces a new product in period $t$. Such a firm sets a price $p_{t}$ in order to maximize:

$$
\Pi\left(\alpha_{t}\right)=\arg \max _{p_{t}} \alpha_{t}\left(1-\bar{r}_{t}\right) p_{t}-F+\delta^{2} \Pi\left(\alpha_{t+2}\right)
$$

where $\Pi\left(\alpha_{t+2}\right)$ is the continuation value at the beginning of period $t+2$, precisely when this firm will be called to produce again. Since along the equilibrium path we have $\left(\bar{r}^{d}, p^{d}\right)$, then $\alpha_{t+2}=1-(1-\mu)\left(1-\bar{r}^{d}\right) \alpha_{t+1}=$ $1-(1-\mu)\left(1-\bar{r}^{d}\right)+(1-\mu)^{2}\left(1-\Delta^{d}-p_{t}\right) \alpha_{t}$. Note that, in this case, the intertemporal demand effect works in the opposite direction than in the monopoly 
case. Now a firm finds it optimal to set a price below the level that maximizes current profits, since a lower $p_{t}$ implies a lower $\alpha_{t+1}$ (which generates a negative externality to the rival firm), which in turn implies a higher $\alpha_{t+2}$; that is, a larger customer base in the next production period.

Thus, following the same procedure used above we can characterize the constant price equilibrium $\left(p^{d}, \bar{r}^{d}\right)$ and check that $p^{d}<p^{m}, \bar{r}^{d}<\bar{r}^{m}$. In other words, even though firms are transitory monopolist, they still compete intertemporally and as a result they set lower prices.

Finally, note that for a large set of values of $F$ firms will indeed have incentives to introduce a new product every period. In particular, suppose $\mu=0$ and $\delta=1$. Along the equilibrium path the lowest value of $\alpha$ is $\alpha_{1}=$ $1-(1-\mu)\left(1-\bar{r}^{d}\right)$. If a firm deviates at $\alpha_{1}$ and does not produce in period 1 , then in period 3 (its next production period) $\alpha_{3}=\alpha_{1}$. Hence, a deviation is not profitable if $\Pi\left(\alpha_{1}\right) \geq 0$. In other words, provided $F$ is below the long-run profit flow, then an equilibrium with production every period does exist. Thus, competition would tend to reinforce the tendency towards excessively frequent introduction of new varieties.

Third, there is the issue of the benefits of coordination in the context of fixed prices. It is well known that in some markets prices respond very little to current market conditions. This is the case, for instance in the movie-theatre industry (Orbach and Einav, 2007). It is easy to check that in a duopoly model with an exogenous price there exists two types of equilibria: one where each firm plays a mixed strategy and the other where the two firms alternate in production. In this case, the social value of coordination (moving from the first to the second type of equilibrium) is clearly positive. On the one hand, firms benefit because they avoid the duplication of fixed costs and jump from a zero expected profit equilibrium to one with generically positive profits. On the other hand, consumers also benefit because they avoid the coordination failure consisting of not having the product available in some periods.

It is precisely in the movie theatre industry where major Hollywood studios seem to continuously play a game of timing with the releases of their close substitutes. Einav (2009) has shown that box office revenues would increase if 
distributors did not cluster their releases so much. In a similar vein, Corts (2001) has shown that when two similar films are both jointly produced and distributed, then they are released further apart than in the case they have neither a producer nor a distributor in common. This suggests integrated structures internalize negative externalities and set schedules in order to maximize joint profits. By spreading opening times more evenly they are able to increase total demand and profits. One reason that might explain why the timing of releases is important (extensively discussed in this empirical literature) is the sharp seasonality of US demand for movies exhibited in theatres. That is, the optimal release date must trade off the benefits from releasing the movie when demand is at its peak with the costs of doing it close to a rival movie. A second possible reason behind the timing game, which empirical researchers may want to consider in the future, is transitory saturation. If the second reason turns out to be sufficiently relevant then, according to our discussion, coordination and information sharing are likely to benefit not only producers but also consumers.

\section{References}

Adda, J. and Cooper, R. (2000), Balladurette and Juppette: A Discrete Analysis of Scapping Subsidies, Journal Political Economy 108(4), 778-806.

Armstrong, M. (2006), Recent Developments in the Economics of Price Discrimination, Chapter 4 in Advances in Economics and Econometrics: Theroy and Applications, Ninth World Congress of the Econometric Society, vol II. Eds Blundell, Newry and Persson. Cambridge University Press.

Becker, G. (1991), A note on Restaurant Pricing and Other Examples of Social Influences on Price, Journal of Political Economy 99 (5), 1109-1116.

Bond, E. and Samuelson, L. (1984), Durable good monopolies with rational expectations and replacement sales, Rand Journal of Economics 15 (3), 336-345.

Chen, Y. and Riordan, M. (2007), Price and variety in the spokes model, Economic Journal 117, 897-921.

Choi, J. (1993), Network Externality, Compatibility Choice, and Planned Obsolescence, Journal of Industrial Economics 42 (2), 167-182.

Corts, K. (2001), The strategic effects of vertical market structure: com- 
mon agency and divisionalization in the US motion picture industry, Journal of Economics and Management Strategy 10 (4), 509-528.

De Meza and von Ungern-Sternberg (1982), Monopoly, product diversity and welfare, Regional Science and Urban Economics 12, 313-324.

Dixit, A. and Stiglitz, J. (1977), Monopolistic competition and optimal product diversity, American Economic Review 67 (3), 297-308.

Driskill, R. (1997), Durable-Goods Monopoly, Increasing Marginal Cost and Depreciation, Economica 64, 137-154.

Einav, L. (2009), Not all rivals look alike: estimating an equilibrium model of the release date timing game, Economic Inquiry , 1-22.

Einav, L. (2007), Seasonality in the US motion picture industry, Rand Journal of Economics 38(1), 745-788.

Ellison, G. and D. Fudenberg (2000), The neo-Luddite's lamment: excessive upgrades in the software industry, Rand Journal of Economics 31 (2), 253-272.

Fishman, A. and R. Rob (2000), Product innovation by a durable-good monopoly, Rand Journal of Economics 31 (2), 237-252.

Krieder, R. and Ch. Weinberg (1998), Competitive Dynamics and the Introduction of New Products: The Motion Picture Timing Game, Journal of Marketing Research 35, 1-15.

Orbach, B. and L. Einav (2007), Uniform prices for differentiated goods: The case of the movie-theater industry, International Review of Law and Economics 27, 129-153.

Salop, S. (1979), Monopolistic competition with outside goods, Bell Journal of Economics 10 (1), 141-156.

Spence, M. (1976), Product selection, fixed costs and monopolistic competition, Review of Economic Studies 43 (2), 217-235.

Waldman, M. (1993), A new perspective on planned obsolescence, Quarterly Journal of Economics 108, 273-283. 


\section{Appendix}

\subsection{Proof of Lemma 1}

We apply Bellman's principle: choose $\bar{r}_{t}$ in order to maximize:

$$
W\left(\alpha_{t}\right)=\frac{\alpha_{t}}{2}\left(1-\bar{r}_{t}^{2}\right)-F+\delta W\left(\alpha_{t+1}\right)
$$

The first order condition of an interior solution (second order condition holds) is:

$$
\bar{r}_{t}=z W^{\prime}\left(\alpha_{t+1}\right)
$$

By the envelop theorem:

$$
W^{\prime}\left(\alpha_{t}\right)=\frac{1}{2}\left(1-\bar{r}_{t}^{2}\right)-z W^{\prime}\left(\alpha_{t+1}\right)\left(1-\bar{r}_{t}\right)
$$

Combining the two previous equations we can write:

$$
W^{\prime}\left(\alpha_{t}\right)=\frac{1}{2}\left(1-\bar{r}_{t}\right)^{2}
$$

Hence, the optimal policy must satisfy:

$$
\bar{r}_{t-1}=\frac{z}{2}\left(1-\bar{r}_{t}\right)^{2}
$$

The stationary solution of this difference equation is:

$$
\bar{r}^{*}=\frac{1+z-\sqrt{1+2 z}}{z}
$$

All the trajectories except $\bar{r}_{0}=\bar{r}^{*}$ are explosive, and hence the unique solution to the optimization problem is $\bar{r}_{t}=\bar{r}^{*}$.

\subsection{Proof of Lemma 2}

Since $\alpha_{0}=1$ then it is obvious that $\gamma_{0}=1$. Let us now consider an arbitrary value of $N, \infty>N>1$. Since, $\alpha_{N+1}=1$, from equation (17)) this implies that $\bar{r}_{N-1}=0$ and $\bar{r}_{t}$ is obtained recursively from equation (18) for $0 \leq t \leq N-2$. Also, note that since $\alpha_{0}=1$, then $0<\alpha_{t}<1$ for $0<t \leq N-1$.

First of all, we show that for any $N, \infty>N>1, \frac{d W^{N}\left(\alpha_{0}\right)}{d \alpha_{0}} \in\left(0, \frac{1}{2}\right)$. In period $t, 0 \leq t \leq N-2$, we have:

$$
W\left(\alpha_{t}\right)=\alpha_{t} R\left(\bar{r}_{t}\right)-F+\delta W\left(\alpha_{t+1}\right)
$$

where $\alpha_{t+1}=1-(1-\mu)\left(1-\bar{r}_{t}\right) \alpha_{t}$. Hence,

$$
\frac{d W\left(\alpha_{t}\right)}{d \alpha_{t}}=R\left(\bar{r}_{t}\right)-z\left(1-\bar{r}_{t}\right) \frac{d W\left(\alpha_{t+1}\right)}{d \alpha_{t+1}}
$$


Let us consider the case $t=N-1$. In this case $\frac{d W\left(\alpha_{N}\right)}{d \alpha_{N}}=0$, since there is no production in period $N$. Moreover, $\bar{r}_{N-1}=0$. As a result, $\frac{d W\left(\alpha_{N-1}\right)}{d \alpha_{N-1}}=\frac{1}{2}$. For an arbitrary $t, 0 \leq t \leq N-2$, if $\frac{d W\left(\alpha_{t+1}\right)}{d \alpha_{t+1}} \geq 0, \frac{d W\left(\alpha_{t}\right)}{d \alpha_{t}}<R\left(\bar{r}_{t}\right) \leq \frac{1}{2}$. Finally, if $\frac{d W\left(\alpha_{t+1}\right)}{d \alpha_{t+1}} \leq \frac{1}{2}, \frac{d W\left(\alpha_{t}\right)}{d \alpha_{t}}>0$.

Suppose that in period 0 it is optimal to follow option $N, \infty>N>1$. This implies that in period $N-1$ option 1 is preferred to option $N$. That is,

$$
W^{1}\left(\alpha_{N-1}\right) \geq W^{N}\left(\alpha_{N-1}\right)
$$

where $\alpha_{N-1}<1$. Then since $\frac{1}{2}=\frac{d W^{1}\left(\alpha_{N-1}\right)}{d \alpha_{N-1}}>\frac{d W^{N}\left(\alpha_{N-1}\right)}{d \alpha_{N-1}}$, it must be the case that:

$$
W^{1}(1) \geq W^{N}(1)
$$

And we reach a contradiction. Therefore, at $\alpha_{0}=1$, the only optimal options are $N=1$, and $N=\infty$.

\subsection{Proof of Lemma 5}

The proof is analogous to that of Lemma 2. Applying Bellman's principle:

$$
\Pi\left(\alpha_{t}\right)=\arg \max _{p_{t}}\left\{\alpha_{t}\left(1-\bar{r}_{t}\right) p_{t}-F+\delta \Pi\left(\alpha_{t+1}\right)\right\}
$$

where $\alpha_{t+1}=1-(1-\mu)\left(1-\bar{r}_{t}\right) \alpha_{t}$, and $\bar{r}_{t}$ is a linear function of $p_{t}$ with slope equal to 1 .

From the first order condition of this optimization problem, we obtain:

$$
p_{t}=1-\bar{r}_{t}+z \Pi^{\prime}\left(\alpha_{t+1}\right)
$$

Using the envelop theorem:

$$
\Pi^{\prime}\left(\alpha_{t}\right)=\left(1-\bar{r}_{t}\right)\left[p_{t}-z \Pi^{\prime}\left(\alpha_{t+1}\right)\right]=\left(1-\bar{r}_{t}\right)^{2}>0
$$

Fix $N, 1<N<\infty$. In period $t=N-1$, which is the period that preceeds the no production period, $\bar{r}_{N-1}=p_{N-1}$ and $\Pi^{\prime}\left(\alpha_{N}\right)=0$. As a result, $p_{N-1}=$ $\bar{r}_{N-1}=\frac{1}{2}$, and $\Pi^{\prime}\left(\alpha_{N-1}\right)=\frac{1}{4}$.

For an arbitrary $t, 0 \leq t \leq N-2, \bar{r}_{t}>p_{t}=1-\bar{r}_{t}+z \Pi^{\prime}\left(\alpha_{t+1}\right)$, which implies that $\bar{r}_{t}>\frac{1}{2}$. Hence, $\Pi^{\prime}\left(\alpha_{t}\right)<\frac{1}{4}$.

Suppose that in period 0 the firm finds it optimal to follow option $N, \infty>$ $N>1$. This implies that in period $N-1$ option 1 is preferred to option $N$. That is,

$$
\Pi^{1}\left(\alpha_{N-1}\right) \geq \Pi^{N}\left(\alpha_{N-1}\right)
$$

where $\alpha_{N-1}<1$. Then since $\frac{1}{4}=\frac{d \Pi^{1}\left(\alpha_{N-1}\right)}{d \alpha_{N-1}}>\frac{d \Pi^{N}\left(\alpha_{N-1}\right)}{d \alpha_{N-1}}$, it must be the case that:

$$
\Pi^{1}(1) \geq \Pi^{N}(1)
$$

And we reach a contradiction. Therefore, at $\alpha_{0}=1$, the only optimal options for the firm are $N=1$, and $N=\infty$. 


\subsection{Proof of Result 1}

A type I equilibrium exists if and only if the following conditions hold:

(i) $\Pi^{\infty}(\bar{\alpha})=\delta \Pi^{\infty}(1)$, which is equivalent to $\bar{\alpha}=F A^{m}$.

(ii) $\bar{\alpha} \leq \bar{r}^{m}$

(iii) The firm does not have incentives to deviate for any $\alpha_{t}$.

Condition (i) indicates that at $\alpha_{t}=\bar{\alpha}$ the firm is indifferent between producing and not producing. Condition (ii) means that along the equilibrium path the lowest value of $\alpha$ is $\alpha_{1}=1-(1-\mu)\left(1-\bar{r}^{m}\right)$, and $\alpha_{1}$ cannot be lower than $\bar{\alpha}$. Conditions $(i)$ and $(i i)$ together imply that $F \leq \frac{\bar{r}^{m}}{A^{m}}=0.0919$.

Let us now consider possible deviations. If $\alpha_{t} \geq \bar{\alpha}$, the optimal deviation may consist of setting a price equal to $\frac{1}{2}$ or equal to $\bar{p}\left(\alpha_{t}\right)$. If $\bar{p}\left(\alpha_{t}\right)$ is higher than $\frac{1}{2}$, then the optimal deviation will consist of setting a price equal to $\frac{1}{2}$ (such a price will be interpreted by consumers as signaling that the next production period is two periods away). However, if $\bar{p}\left(\alpha_{t}\right)$ is lower than $\frac{1}{2}$, then the optimal deviation price is slightly below $\bar{p}\left(\alpha_{t}\right)$, in order to signal that in the next period there will be no production.

Note that $\bar{p}(1)=\bar{\alpha}-\Delta=A^{m} F-\Delta \geq \frac{1}{2}$ if and only if $F \geq 0.0863$. But in this case, it is clear that the firm has incentives to deviate at $\alpha_{t}=1$, since it makes $\frac{\frac{1}{4}-F}{1-\delta^{2}} \geq \frac{1}{A^{m}(1-\delta)}-\frac{F}{1-\delta}$. In fact, the last inequality holds for any $F \geq 0.0502=F^{m}$. Thus, a necessary condition for the existence of a type I equilibrium is that $F<0.0863$.

If $F<0.0863$ at $\alpha_{t}=1$, whenver the firm deviates, then it sets $p_{t}=\bar{p}(1)$. Such a deviation will not be profitable whenever the following condition holds:

$$
\frac{\bar{p}(1)[1-\bar{p}(1)]-F}{1-\delta^{2}} \leq \frac{1}{(1-\delta) A^{m}}-\frac{F}{1-\delta}
$$

Since $\bar{p}(1)=A^{m} F-\Delta$, and if we take the limit of $\delta$ going to 1 , then this condition becomes $F \leq 0.0669$.

Incentives to deviate are weaker if $\alpha_{t}<1$. Along the equilibrium path $\frac{d \Pi^{\infty}\left(\alpha_{0}\right)}{d \alpha_{0}}=\frac{1}{A^{m}}$. If the firm deviates in period 0 , then $\alpha_{2}=1$; hence the expected profits in case of a deviation can be written as $\Pi^{D}\left(\alpha_{0}\right)=\alpha_{0} \bar{p}\left(\alpha_{0}\right)\left[1-\bar{p}\left(\alpha_{0}\right)\right]+$ $\delta^{2} \Pi^{1}(1)$. Therefore, $\frac{d \Pi^{D}\left(\alpha_{0}\right)}{d \alpha_{0}}=\bar{p}\left(\alpha_{0}\right)^{2}-2(1-\Delta) \bar{p}\left(\alpha_{0}\right)+1-\Delta>\frac{1}{4}>\frac{1}{A^{m}}$.

\subsection{Poof of Result 2}

Suppose that $\alpha_{t} \in[\bar{\alpha}, 1]$. If consumers expect that in the next period $\alpha_{t+1} \leq \underline{\alpha}$ then their optimal consumption decision is $\bar{r}_{t}=p_{t}$. The condition that supports these believes, given consumers' behavior, is $1-\left(1-p_{t}\right) \alpha_{t} \leq \underline{\alpha}$. That is, $p_{t} \leq 1-\frac{1-\underline{\alpha}}{\alpha_{t}}$. On the contrary, if $p_{t}>1-\frac{1-\underline{\alpha}}{\alpha_{t}}$ then consumers will rationally expect that either $\alpha_{t+1} \in(\underline{\alpha}, \bar{\alpha})$ or $\alpha_{t+1} \in[\bar{\alpha}, 1]$. In the former case, the optimal consumer strategy is $\bar{r}_{t}=p_{t}+\frac{1}{16}$, and the condition is $p_{t} \in\left(1-\frac{1-\underline{\alpha}}{\alpha_{t}}, \frac{15}{16}-\frac{1-\bar{\alpha}}{\alpha_{t}}\right)$. Finally, if $p_{t} \geq \frac{15}{16}-\frac{1-\bar{\alpha}}{\alpha_{t}}$ then the optimal cosumer strategy is $\bar{r}=\frac{1}{8}+p_{t}$. 
Suppose that $\alpha_{t} \in[0, \bar{\alpha})$. If consumers expect that in the next period $\alpha_{t+1} \geq$ $\bar{\alpha}$ then their optimal consumption behavior is $\bar{r}=\frac{1}{8}+p_{t}$. The condition that supports these believes, given consumers' behavior, is $1-\left(\frac{7}{8}-p_{t}\right) \alpha_{t} \geq \bar{\alpha}$. That is, $p_{t} \geq \frac{7}{8}-\frac{1-\bar{\alpha}}{\alpha_{t}}$. On the contrary, if $p_{t}<\frac{7}{8}-\frac{1-\bar{\alpha}}{\alpha_{t}}$ then consumers will expect that either $\alpha_{t+1} \in(\underline{\alpha}, \bar{\alpha})$ or that $\alpha_{t+1} \in[0, \underline{\alpha}]$. In the former case, the optimal consumer strategy is $\bar{r}_{t}=p_{t}+\frac{1}{16}$, and the associated condition is $p_{t} \in\left(\frac{15}{16}-\frac{1-\underline{\alpha}}{\alpha_{t}}, \frac{7}{8}-\frac{1-\bar{\alpha}}{\alpha_{t}}\right)$. Finally, if $p_{t} \leq \frac{15}{16}-\frac{1-\underline{\alpha}}{\alpha_{t}}$ then the optimal consumer strategy is $\bar{r}_{t}=p_{t}$.

If $\alpha_{t} \in(\underline{\alpha}, \bar{\alpha})$ the firm plays $\gamma_{t}=1$ and $p_{t}=\frac{5}{8}$, expecting that $\alpha_{t+1} \geq \bar{\alpha}$. Consumers will share the same expectations if $\frac{5}{8} \geq \frac{7}{8}-\frac{1-\bar{\alpha}}{\alpha_{t}}$; that is, $\frac{1-\bar{\alpha}}{\alpha_{t}} \geq \frac{1}{4}$. Hence, $\bar{\alpha} \leq \frac{4}{5}$.

If $\alpha_{t} \in[\bar{\alpha}, 1]$, the firm plays $\gamma_{t}=1$ and $p_{t}=\frac{1}{2}$, expecting that $\alpha_{t+1} \leq \underline{\alpha}$. Consumers will share the same expectations if $\frac{1}{2} \leq 1-\frac{1-\underline{\alpha}}{\alpha_{t}}$; that is, $\frac{1-\underline{\alpha}}{\alpha_{t}} \leq \frac{1}{2}$. This implies that $\underline{\alpha} \geq 1-\frac{\bar{\alpha}}{2}$.

Finally, we have to check that the firm does not have incentives to deviate. If $\alpha_{t} \in(\underline{\alpha}, \bar{\alpha})$, the firm obtains an equilibrium payoff of:

$$
\alpha_{t} \frac{1}{4} \frac{5}{8}-F+\delta\left(1-\frac{1}{4} \alpha_{t}\right) \frac{1}{4}-\delta F+\delta^{3} \Pi^{1}(1)
$$

If instead the firm chooses not to produce then it gets $\delta \Pi^{1}(1)$. At $\alpha_{t}=\underline{\alpha}$ the firm must be indifferent between these two options. Hence,

$$
\underline{\alpha}=\frac{32}{3} F
$$

The widest range of parameters that sustain a type II equilibrium is $\bar{\alpha}=\frac{4}{5}$, which implies that $\underline{\alpha} \geq \frac{3}{5}$. Since $\underline{\alpha}$ mult lie in the interval $\left[\frac{3}{5}, \frac{4}{5}\right]$ then a type II equilibrium exist only if $F \in[0.0562,0.0750]$.

Still in the case $\alpha_{t} \in(\underline{\alpha}, \bar{\alpha})$, the firm could consider a price in the range $\left(\frac{15}{16}-\frac{1-\underline{\alpha}}{\alpha_{t}}, \frac{7}{8}-\frac{1}{5 \alpha_{t}}\right)$ and face a consumer behavior described by $\bar{r}_{t}=p_{t}+\frac{1}{16}$. 
Figure 1

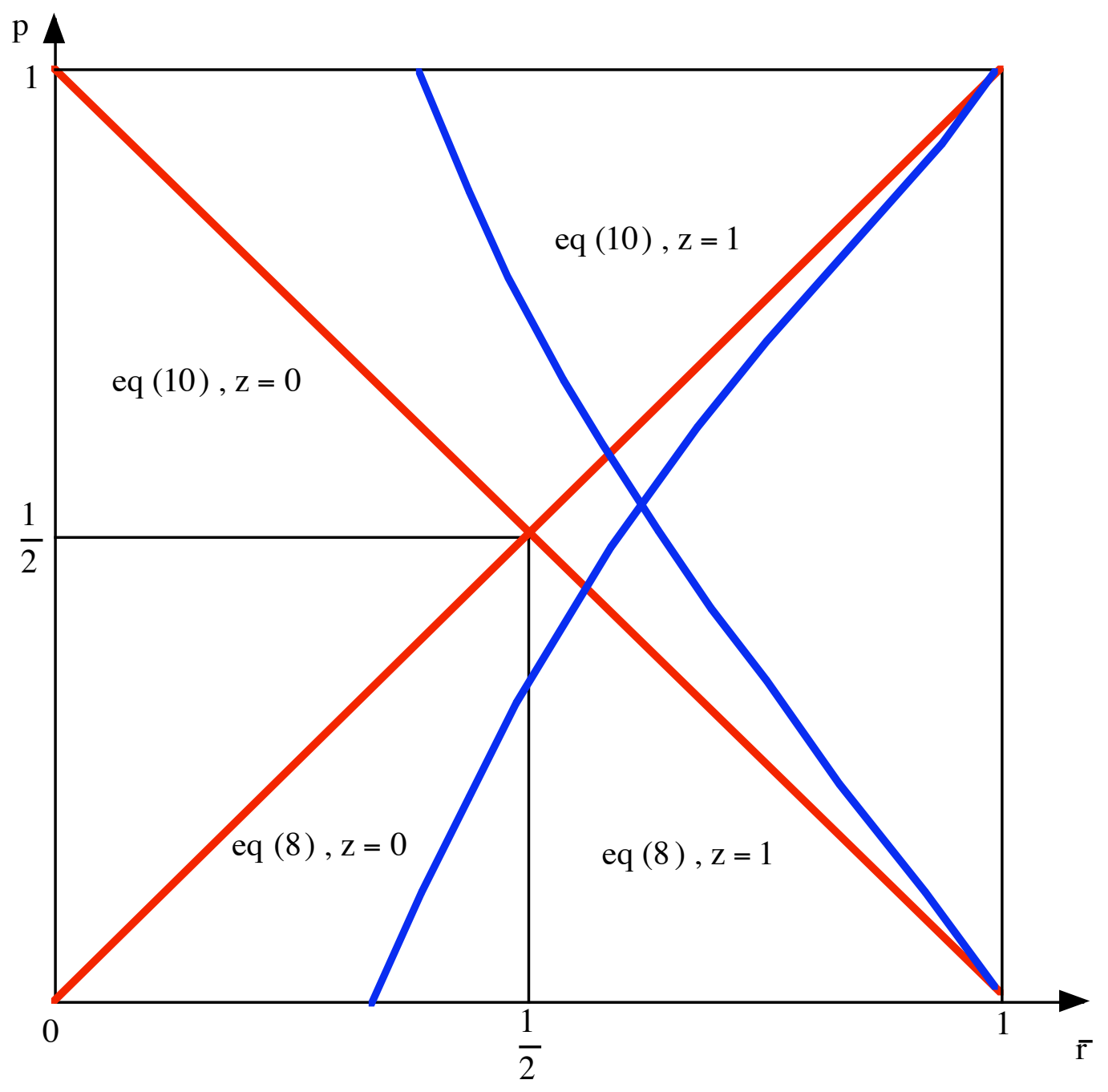

